\title{
Low-Intensity Pulsed Ultrasound-Generated Singlet Oxygen Induces Telomere Damage Leading to Glioma Stem Cell Awakening From Quiescence
}

\section{Sirong Song}

Tianjin Medical University

Dongbin Ma

Tianjin Medical University

Lixia Xu

Tianjin Huanhu Hospital

Qiong Wang

Tianjin Huanhu Hospital

Lanxiang Liu

: First Hospital of Qinhuangdao

Xiaoguang Tong

Tianjin Huanhu Hospital

Hua Yan ( $\nabla$ yanhua20042007@sina.com )

Tianjin Huanhu Hospital

Research

Keywords: GSC, LIPUS, telomere, singlet oxygen, cytochrome

Posted Date: February 8th, 2021

DOl: https://doi.org/10.21203/rs.3.rs-157904/v1

License: (c) (1) This work is licensed under a Creative Commons Attribution 4.0 International License. Read Full License 


\section{Abstract}

Background: Cancer stem cells, quiescent and drug-resistant, have become a therapeutic target. Lowintensity pulsed ultrasound (LIPUS), a new noninvasive physical device, promotes pluripotent stem cell differentiation and is mainly applied in tissue engineering but rarely in oncotherapy. We explored the effect and mechanism of LIPUS on glioma stem cell (GSC) expulsion from quiescence.

Methods: Immunofluorescence staining and flow cytometry were used to detect changes of stem hallmarkers. RT-PCR results showed the gene expression levels of stem-related transcription factors. Brud and RNA-seq were performed for cell cycle analysis. Western blotting showed different expressions of key point proteins. Telomeres damage was found by FISH and IF-FISH. Fluorescence detection was used for mitochondrial membrane potential assay and singlet oxygen detection. Tumor xenograft and immunohistochemical staining were performed to confirm the role of low intensity pulsed ultrasound.

Results: We found that LIPUS led to attenuated expression of GSC hallmarks, promoted GSC escape from G0 quiescence, and significantly weakened the stemness-related Wnt and Hh pathways. Next, Interestingly, LIPUS transferred sonomechanical energy into recombinant cytochrome C and B5 proteins in vitro, which converted oxygen molecules into singlet oxygen, triggering a telomere crisis. The results in vivo and in vitro confirmed that LIPUS enhanced GSC sensitivity to temozolomide.

Conclusion: These results demonstrated that LIPUS "wakes up" GSCs to improve chemotherapy by transferring energy to cyt families and leading to telomere crisis.

\section{Background}

Cancer stem cells (CSCs) are a special cancer cell population with the capacity of self-renewal, proliferation and multilineage differentiation, similar to stem cells(1-5). CSCs can promote tumorigenesis and recurrence through self-renewal and differentiation. In particular, CSCs begin to move out of quiescence, thus contributing to cancer propagation. They have also demonstrated an enhanced capacity for therapeutic resistance, immune evasion, invasion, and metastasis(6-8). Different from dividing cancer cells with a high metabolism, they generally remain dormant(9). Given that conventional anticancer therapies preferentially target dividing cells, CSCs are resistant to such treatments, with those remaining after the elimination of bulk cancer cells potentially giving rise to disease relapse and metastasis as they re-enter the cell cycle after a period of latency. Targeting the switch between quiescence and proliferation in CSCs is therefore a potential strategy for preventing the reinitiation of malignancy.

Ultrasound has been widely applied in disease diagnosis(10) and adjuvant therapy(11). Low-intensity pulsed ultrasound (LIPUS) has low energy intensity $\left(<3 \mathrm{w} / \mathrm{cm}^{2}\right)$ and outputs energy in the form of an impulse wave. It has been widely applied in regenerative medicine because of its noninvasiveness and few side effects, such as promoting fracture healing(12), accelerating tissue regeneration(13), enhancing thrombolysis(14) and nerve regeneration(15). LIPUS could improve the osteogenic differentiation of human mesenchymal stem cells(16) and guarantee the maintenance of the osteogenic committed 
fraction(13). However, there are few studies about the regulatory effect and the mechanism of LIPUS on CSCs. Based on the role of LIPUS in human mesenchymal stem cells, we hypothesized that LIPUS could "wake up" dormant CSCs to accelerate CSC expulsion from quiescence. The main purpose of our project was to explore whether LIPUS "wakes quiescent CSCs up", decreases the expression of CSC stem cellrelated characteristics, and enhances the sensitivity of chemoradiotherapy to promote the killing role of chemoradiotherapeutic drugs on CSCs to provide new ideas for prolonging the survival of patients.

More interestingly, the mechanism by which ultrasound exerts its energetic effects at the cellular level is still unclear. The CSC energy source relies primarily on mitochondrial respiration, which is distinct from aerobic glycolysis in tumor cells(17-19). It has been reported that singlet oxygen $\left({ }^{1} \mathrm{O}_{2}\right)$ produced by mitochondria can selectively damage telomeres $(20,21)$. Telomeres are involved in CSC stemness $(22,23)$. Accordingly, we explored whether LIPUS produces ${ }^{1} \mathrm{O}_{2}$ from the mitochondria, leading to a telomere crisis and dysfunction. In addition, as sonosensitizers, cytochrome B5 and C can absorb energy and undergo electronic transitions in response to LIPUS treatment, which are excited from a low-energy state to a highenergy state, and then transfer the energy to the ground state oxygen molecules to release ${ }^{1} \mathrm{O}_{2}$.

\section{Materials And Methods}

\section{Cell culture and dedifferentiation}

U87 glioblastoma cells were obtained from the Cell Resource Center, Institute of Basic Medicine, Chinese Academy of Medical Sciences. U87 cells were continuously cultured in MEM medium (Gibco, USA) with $10 \%$ fetal bovine serum (Gibco, USA) and 1\% penicillin/streptomycin (Thermo Fisher, USA) at $37^{\circ} \mathrm{C}$ in humidified chambers under $5 \% \mathrm{CO}_{2}$. And GSCs from U87 cells were cultured in a serum-free cancer stem cell medium.

\section{LIPUS stimulating}

LIPUS is sinusoidal ultrasound with a frequency of $1.5 \mathrm{MHz}$. Cells were sonicated at $300 \mathrm{MW} / \mathrm{cm}^{2}$ for the $1.0 \mathrm{kHz}$ system using $200 \mathrm{~ms}$ pulses of ultrasound with $800 \mathrm{~ms}$ spaced in between each pulse. LIPUS was performed once a day for $20 \mathrm{~min}$ at the same time per day for 24,48 , and $72 \mathrm{~h}$.

\section{Immunofluorescence staining}

GSCs were plated onto poly-L-lysine-coated glass coverslips in DMEM/F12 for $1 \mathrm{~h}$, washed three times with PBS and then fixed with 4\% paraformaldehyde (Sigma, USA) for $20 \mathrm{~min}$. Then the cells were incubated with antibodies against CD133 (Sigma, USA) and Nestin (Sigma, USA) at $4^{\circ} \mathrm{C}$ overnight, washed with PBS and incubated with secondary antibodies at $37^{\circ} \mathrm{C}$ for $1 \mathrm{~h}$. The nuclei were stained with DAPI. The slides were examined using confocal microscopy.

\section{Cell Viability Assay}


CCK-8 assays were applied to analyze the influence of LIPUS on the proliferation of the GSCs. CCK-8 assays were followed manufacturer's instructions.

\section{Annexin V/7-AAD Apoptosis Assay}

Annexin V/7-AAD Apoptosis Assays were applied to analyze the influence of LIPUS on the apoptosis of GSCs. Manufacturer's instructions were followed.

\section{5-bromodeoxyuridine (BrdU) Assay}

BrdU assays were applied to analyze the influence of LIPUS on the cell cycle of GSCs. Cells were plated at a density of $2 \times 10^{6}$ per 6-well plate. BrdU (BD Biosciences, USA) was added to the cell suspensions. Then, the cells were cultured at $37^{\circ} \mathrm{C}$ for $1 \mathrm{~h}$ and then analyzed using a BD Caliber flow cytometer.

\section{Fluorescence activated cell sorting (FACS)}

GSCs were collected and resuspended in $100 \mu$ antibody incubation buffer. Anti-CD133 antibody was added and incubated on ice for $30 \mathrm{~min}$. After centrifugation and resuspension, the cells were fixed and membrane-perforated. Then, $20 \mu \mathrm{l}$ anti-Nestin antibody was added. The expression rates were detected and calculated using a BD Caliber flow cytometer.

\section{8. qPCR (Quantitative PCR)}

Genomic DNA was extracted using a DNA extraction kit (Biogama,USA) for qPCR. qPCR was performed on the Roche Lightcycler II using telomeric primers (Tel) and primers for a reference control gene (the human 36B4 single-copy gene). The qPCR primers are presented in supplementaryTable 1.

\section{RT-PCR (Reverse Transcription-PCR)}

Total RNA was extracted using TRIzol (Invitrogen Life Technologies, Carlsbad, CA, USA) according to the manufacturer's instructions. RNA was reverse transcribed using an RT-PCR kit (Takara Bio, Beijing, China). The RT-PCR primers are presented in Supplementary Table 1.

\section{Metaphase Spreads and Telomere Fluorescence in Situ Hybridization (FISH)}

\section{Analysis.}

FISH was used to detect the telomere loss. GSCs were collected and treated with $10 \mu \mathrm{g} / \mathrm{ml}$ colcemid for $20 \mathrm{~h}$, incubated with $75 \mathrm{mM} \mathrm{KCl}$ for $30 \mathrm{~min}$ at $37^{\circ} \mathrm{C}$, prefixed for $5 \mathrm{~min}$ and then fixed for $1 \mathrm{~h}$ with methanol: glacial acetic acid (3:1). The cells were dropped onto water-coated slides to spread the metaphase chromosomes. Then slides were stained with the PNA probe following manufacturer's protocol. Images were caught by a Nikon Ti inverted fluorescence microscope. The images were analyzed using NIS Elements Advanced Research software. 


\section{Immunofluorescence and telomere fluorescence in situ hybridization (IF-FISH)}

Telomere damage was analyzed though IF-FISH. GSCs were plated onto poly-L-lysine-coated glass coverslips in DMEM/F12 for $1 \mathrm{~h}$ and then fixed with $4 \%$ paraformaldehyde for $20 \mathrm{~min}$. The cells were permeabilized with $0.2 \%$ Triton X-100 (Sigma, USA) and dehydrated through graded alcohol. The coverslips were hybridized with the PNA probe, then 53bp1 antibody and second antibody following manufacturer's protocol. Images were acquired on a Nikon Ti inverted fluorescence microscope. The images were deconvoluted and analyzed using NIS Elements Advance research software. The foci counts were exported to Excel for analysis.

\section{Western blotting}

Proteins were extracted from GSCs treated with LIPUS using RIPA lysis buffer and PMSF (Solarbio Co., Beijing, China) following the manufacturer's protocols. The proteins were separated by SDS-PAGE, transferred to a nitrocellulose membrane, blocked with bovine serum albumin and then incubated with primary antibodies against Gli1, PTCH, $\beta$-actin, Wnt3, Wnt, $\beta$-cateninPLK1, CDC2 and CyclinB (CST, USA) at $4^{\circ} \mathrm{C}$ overnight. After the membrane was incubated with the goat anti-rabbit/mouse IgG HRP secondary antibody (CST, USA) for $90 \mathrm{~min}$, chemiluminescence membrane exposure and enhancement were performed.

\section{Mitochondrial Membrane Potential Assay}

Mitochondrial Membrane Potential Assay was detected with the JC-1 detection kit (Dojingo Molecular Technology). The JC-1 kit manufacturer's instruction was followed.

\section{Singlet oxygen $\left(\mathrm{O}_{2}\right)$ Detection}

Singlet oxygen fluorescence intensity was detected by the enzyme labeler and GSCs were photographed under the confocal microscope following manufacturer's instruction.

\section{Tumor xenograft study}

All experimental protocols were conducted in accordance with national legislation and associated guidelines. BALB-c nude mice (male; grade SPF; 4-6 w, 16-18 g) were purchased from the Experimental Animal Center of Academy of Military Medical Sciences, China. The xenograft tumor volume was calculated according to the formula $V=1 / 6 \pi\left(a b^{2}\right)$, and then the tumor growth curves were plotted.

\section{Immunohistochemistry}

TdT-mediated dUTP nick end labeling (TUNEL) and Ki67 staining at the end of the 16-day experimental period to analyze the apoptosis and proliferation of tumor tissues. Manufacturer's instructions were followed for both methods

\section{Statistical Analysis}


Statistical analyses were performed using SPSS scientific software. The applied methods were Student's t-test for the comparison of two groups and one-way ANOVA for more than two comparisons. Differences with $p$ values less than 0.05 were considered statistically significant.

\section{Results}

\section{LIPUS promotes GSCs to escape from the stem cell state.}

To investigate the effect of LIPUS on GSCs, GSCs derived from U87 cells were induced in vitro and stimulated by LIPUS for 3 consecutive days. Under the microscope at 100× (Fig. 1A), it was found that the ability of stem cell sphere formation was weakened with LIPUS treatment ( $100 \%$ vs $58 \%$ ). The GSC spheres of the control group were large in size with round and smooth edges. Conversely, GSC spheres of the LIPUS group were smaller and uneven in size, with loose cells and less tight intercellular connections than those of the control group, indicating that LIPUS changed the morphology of the tumor spheroids, triggering the production of GSC spheres with extended antennae such as are found in nonstem cancer cells, and promoted GSC to move from a stem cell state to a nonstem cell state.

Next, we used immunofluorescence staining and flow cytometry to detect changes in the expression levels of stem cell surface markers, including CD133 and Nestin protein (Fig. 1B). Fluorescence staining results showed that CD133 and Nestin were highly expressed in the control group, while those in the LIPUS group were significantly decreased. Additionally, the flow cytometry results of Nestin showed that after LIPUS stimulation for $24 \mathrm{~h}, 48 \mathrm{~h}$ and $72 \mathrm{~h}$, the proportion of Nestin-positive GSC spheres decreased from $90.8 \%$ to $57.3 \%$, suggesting that the Nestin-positive CSC spheres decreased gradually, while the Nestin-negative nonstem cell spheres increased gradually (Fig. 1C). The Reverse transcription-polymerase chain reaction (RT-PCR) results showed that the gene expression levels of stem-related transcription factors Nanog, Oct4, and Sox2, and the stem cell markers CD133 and Nestin after LIPUS intervention at different times were all lower than in those of the control group (Fig. 1D). These results suggest that LIPUS induces the loss of stem cell-related characteristics and the differentiation of CSCs.

\section{LIPUS regulates the $\mathrm{G} 2 / \mathrm{M}$ checkpoint and induces apoptosis.}

We used 5-bromodeoxyuridine (Brud) flow cytometry to investigate whether LIPUS is associated with regulation of the GSC cell cycle (Fig. 2A). Compared with the control group, G0/G1 cells were less abundant ( $50 \%$ vs $30 \%$ ), and G2/M cells were more abundant ( $12 \%$ vs $26 \%$ ) in the LIPUS group. These results suggest that LIPUS "woke up" dormant GSCs from the quiescent G0 phase into the active G2/M phase, inducing GSC differentiation. Furthermore, RNA SEQ sequencing was performed in each group of three replicates. A total of 255 differentially expressed genes ( $p$ value $<0.05$, log2FoldChange $>0.0$ ) were detected, including 172 upregulated genes and 83 downregulated genes (Fig. 2B). Next, we carried out Reactome functional enrichment analysis on the differential gene sets. The results showed that 3 of the top 10 enriched pathways were related to cell cycle regulation (Fig. 2C). Then, Reactome pathway research on the differentially expressed genes was carried out (Fig. 2D). The results showed that the differentially expressed genes were also enriched in the cell cycle regulation pathway, and the key 
proteins of the G2/M checkpoint were significantly regulated, including PLK1, CDK1, CyclinB, etc. Meanwhile, the western blotting was preformed to verify this conclusion (Fig. 2E). The expression level of PLK1, CDK1 and CyclinB expression increased significantly with LIPUS treatment. These results suggest that LIPUS promoted mitosis of CSCs from the G0 phase to the G2/M phase.

\section{LIPUS inhibits the Hh/Wnt pathway and reduces the stem cell-related characteristics of CSCs.}

The self-renewal and stemness maintenance of CSCs are regulated by a variety of signaling pathways, and the Hedgehog $(\mathrm{Hh})$ and Wnt/ $\beta$-catenin pathways are widely known. To determine how LIPUS reduced GSC stemness, we found by western blot (Fig. 2F) that the expression of Wnt3, Wnt5 and $\beta$-catenin, the key nodes of Wnt signaling, were significantly downregulated after LIPUS treatment for 24,48 and $72 \mathrm{~h}$. Similarly, the expression levels of Gli1 and PTCH, the key nodes of the Hh signaling pathway, were significantly reduced. These results indicated that LIPUS inhibited the Hh/Wnt pathway to reduce GSC stem cell-related characteristics.

\section{LIPUS shortens the telomere length and leads to telomere damage.}

We detected the telomere length and integrity in GSCs after LIPUS treatment by Quantitative polymerase chain reaction (qPCR) and metaphase spread and telomere fluorescence in situ hybridization (FISH). The telomere length (T/S) of the LIPUS group was significantly shorter than that of the control group, which was approximately $50 \%$ that of the control group (Fig. 3A). The fluorescence intensity of the telomeres in the LIPUS group was significantly weaker. The results of metaphase spread and telomere FISH (Fig. 3B) showed that the average number of telomeres lost in each cell of the control group was 1.71, while it was 6.9 per cell in the LIPUS group, suggesting telomere length shortening and telomere loss due to LIPUS.

Furthermore, we tested whether there was telomere damage in GSCs by Immunofluorescence and telomere fluorescence in situ hybridization (IF-FISH) technology (Fig. 3C). The 53 bp1 is a DNA repair protein for double-stranded breaks, which are known to be recruited to telomeres upon double-strand break formation. Telomere dysfunction-induced focus (TIFs) where telomeres and the 53BP1 fluorescence signal meet indicate telomere damage. There were more fluorescence signal points of TIFs in the LIPUS group, with an average of $3.8 \mathrm{TIFs}$ per cell and $0.57 \mathrm{TIFs}$ per cell in the control group. TIFs in the LIUPS group were significantly higher than those in the control group, indicating that LIPUS caused telomere damage and inhibited the DNA protection function of telomeres.

\section{LIPUS on mitochondrial cytochrome (cyt) produces ${ }^{1} 02$, inducing telomere}

In healthy cells with high mitochondrial membrane potential, JC-1 forms complexes known as Jaggregates with intense red fluorescence. However, in cells with low mitochondrial potential (depolarized), $\mathrm{JC}-1$ remains in the monomeric form, exhibiting green fluorescence. First, we monitored changes in mitochondrial membrane potential. The fluorescence intensity of $\mathrm{JC}-1$ in living cells was detected with the $\mathrm{JC}-1$ detection kit, and the ratio of red/green fluorescence intensity was used to reflect the mitochondrial membrane potential (Fig. 4A). The results showed that the red/green fluorescence intensity 
ratio in the LIPUS group was significantly lower than that in the control group, suggesting that the mitochondrial membrane potential decreased significantly with LIPUS treatment. Thus, LIPUS caused mitochondrial damage and oxidative stress. At $24 \mathrm{~h}, 48 \mathrm{~h}$ and $72 \mathrm{~h}$ after stimulation by LIPUS, the ratio of red/green fluorescence intensity decreased successively (Fig. 4B), indicating that the influence of LIPUS on the mitochondrial membrane potential was positively correlated with the stimulation duration.

It has been reported that ${ }^{1} \mathrm{O}_{2}$ produced by mitochondria can damage telomeres in a targeted manner. Therefore, we used a singlet oxygen detection kit to detect the influence of LIPUS on ${ }^{1} \mathrm{O}_{2}$ production in GSCs (Fig. 4C). The results showed that the fluorescence intensity of probe R in the LIPUS group was higher than that in the control group, indicating that the level of ${ }^{1} \mathrm{O}_{2}$ in the LIPUS group was higher than that in the control group. This suggests that LIPUS increased ${ }^{1} \mathrm{O}_{2}$ production in GSCs. Compared with the treatment with LIPUS for $10 \mathrm{~min}$, the ${ }^{1} \mathrm{O}_{2}$ production at 20 min was improved (Fig. 4D), indicating that the ${ }^{1} \mathrm{O}_{2}$ production was related to the extension of the treatment time with LIPUS.

To further clarify the mechanism of the effect of LIPUS on ${ }^{1} \mathrm{O}_{2}$, we conducted in vitro experiments. We detected ${ }^{1} \mathrm{O}_{2}$ production in pure proteins of recombinant cytochrome $\mathrm{B} 5 \mathrm{a}$ (cyt $\mathrm{B} 5 \mathrm{a}$ ), cytochrome $\mathrm{B} 5 \mathrm{~b}$ (cyt B5b), cytochrome C (cyt C) and cytochrome C1 (cyt C1) after LIPUS treatment. The results showed that the standard fluorescence intensity of probe $\mathrm{R}$ in the cyt B5 group was significantly higher than that in the control group, indicating that ${ }^{1} \mathrm{O}_{2}$ production in the LIPUS group was higher than that in the control group (Fig. 4E). In the cyt $\mathrm{C}$ group, the standard fluorescence intensity of probe $\mathrm{R}$ was higher than that of the control group (Fig. $4 \mathrm{~F}$ ), indicating that the ${ }^{1} \mathrm{O}_{2}$ production in the LIPU group was higher than that of the control group. These results suggested that LIPUS produces ${ }^{1} \mathrm{O}_{2}$ by acting on the cyt B5 family and cyt $\mathrm{C}$ family to trigger intracellular oxidative stress.

\section{LIPUS enhances the chemotherapeutic sensitivity of Temozolomide (TMZ) and increases the cytotoxic effect on GSCs.}

Clear eradication of CSCs is of clinical importance due to their close relationship with the malignant nature of cancer cells, such as tumorigenicity and chemoresistance. Compared with the TMZ group, the growth rate of GSCs in the LIPUS+TMZ group was slower as determined by the cell counting kit-8 (CCK8) assay, and the proportion of GSC apoptotic cells in the LIPUS+TMZ group was higher (28\% vs $17 \%)$ in the Annexin V-APC/7-aminoactinomycin D (7-AAD) assay, suggesting that LIPUS enhanced the cytotoxic effect of TMZ on GSCs (Fig. 5A). To investigate the effect of LIPUS on tumor resistance to chemotherapy, we constructed a nude mouse model of subcutaneous implantation (Fig. 5B). Twenty nude mice were randomly divided into four groups: Ctrl, LIPUS, TMZ and LIPUS+TMZ. After 7 days of tumor implantation, the mice began to receive LIPUS and TMZ treatment. The size of the tumor changed obviously on the 8th day. As shown in the figure, the tumor volume of the LIPUS+TMZ group was significantly smaller than that of the other groups on the 16th day of treatment. The tumor volume of the control group was nearly 3.75 times that of the LIPUS+TMZ group, suggesting that LIPUS+TMZ significantly inhibited tumor 
growth. Importantly, the tumor volume of the TMZ group was almost 1.5 times that of the TMZ+LIPUS group, illustrating that LIPUS enhanced the cytotoxic effect of TMZ and had a negative impact on tumor growth in combination with TMZ.

To thoroughly investigate the effect of LIPUS combined with TMZ on tumor cell growth, tumor tissues were fixed and sectioned for TdT-mediated dUTP nick end labeling (TUNEL) and Ki67 staining at the end of the 16-day experimental period to analyze the apoptosis and proliferation of tumor tissues, respectively. The TUNEL results showed that on average, there were 2 positive cells in each field of vision in the control group, 4.75 in the LIPUS group, 14.75 in the TMZ group, and 21.75 in the LIPUS+TMZ group (Fig. 4C). The Ki-67 results showed that in the control group, the level of Ki-67 expression was the highest, and the positive area accounted for $90 \%$ in each field of vision, $62 \%$ in the LIPUS group, $35 \%$ in the TMZ group, and 15\% in the LIPUS+TMZ group (Fig. 4D). Compared with that in the control group, the tumor size in the LIPUS group was smaller, with higher expression of TUNEL and lower expression of Ki67, suggesting that LIPUS inhibited the growth of tumors in vivo. Compared with the TMZ group, there was a decrease in the tumor size and the expression of Ki67 and an increase in the expression of TUNEL in the LIPUS+TMZ group, indicating that LIPUS enhanced the chemotherapy sensitivity of the glioma to TMZ.

These results showed that LIPUS promoted TMZ inhibition of tumor growth and increased the sensitivity of the tumor to TMZ, which also suggested that LIPUS reduced the resistance of CSCs to chemotherapeutic agents and promoted CSC differentiation.

\section{Discussion}

Cancer stem cells, with the capacity for infinite proliferation and multipotency, play a crucial role in the initiation, progression and recurrence of cancer. Although they account for less than $1 \%$ of the tumor population, they are able to produce most other tumor cells $(2,3,24)$. At present, current therapy regimens, such as chemotherapy and radiation therapy, target dividing tumor cells rather than CSCs. Even though $99 \%$ of most tumor cells are initially killed, the treatment could only "cut branches and leaves" rather than

"eliminate roots". As long as $1 \%$ CSCs are present, tumor recurrence eventually occur(7). CSCs, closely related to tumor invasion, metastasis and chemoradiotherapy tolerance $(8,9)$, have become an important therapeutic target $(25,26)$.

As a novel noninvasive physical device, sonodynamic therapy can reversibly open the blood-brain barrier, enhance the delivery of drugs to the brain, and effectively kill tumors $(27,28)$. In recent years, most researchers have focused on the thermal effect and cavitation of high-intensity focused ultrasound to inhibit the proliferation of cancer $(29,30)$ and to enhance apoptosis when combined with chemotherapy agents $(31,32)$. In contrast to the strong cytotoxicity of high-intensity focused ultrasound on tumors, LIPUS possesses the ability to promote fracture healing(12), accelerate regeneration(13), and to enhance thrombolysis(14) and nerve regeneration(15). More recently, LIPUS has been reported to promote myoblast alignment for tissue engineering and the differentiation of mesenchymal stem cells in the treatment of spinal cord injury $(16,33)$. Compared with tumor cells, cancer stem cells are more sensitive to 
sonication interventions(34). Fei Qu et al. reported that sonodynamic therapy induces glioma cell apoptosis(29). There are few reports about the regulatory effect of LIPUS on CSCs. Naturally, we supposed that LIPUS could promote CSC expulsion from quiescence, similar to its role in mesenchymal stem cells.

CSCs, mostly in the G0 phase of the cell cycle, remain quiescent for a long time with a very low metabolism. DNA replication, transcription, and translation are almost suspended. Therefore, conventional chemotherapeutic agents targeting dividing cells cannot eliminate them even though CSCs are present in small quantities and have inadequate proliferation $(4,5)$. CSCs have strong DNA repair ability with active telomerase and drug resistance and are regulated by a series of signaling pathways, such as the Wnt pathway, Notch pathway and Hedgehog $(\mathrm{Hh})$ pathway. In this experiment, the expression of Gli1 and PTCH, the key nodes of the Hh signaling pathways related to self-replication and stemness maintenance of tumor stem cells, were clearly downregulated. Consistently, the expression of Wnt signaling pathways, including Wnt3, Wnt5 and $\beta$-catenin, were significantly inhibited. In addition, the gene expression levels of stem cell-related transcription factors Nanog, Oct4, and Sox2 $(35,36)$ and the stem cell markers CD133 and Nestin were decreased. In contrast to the adherent growth of most tumor cells, CSCs have a tendency to form tumor spheres in suspension(37). Mature CSC spheres, round and smooth, with tightly packed cells, are formed in suspension. With LIPUS treatment, the sphericity of GSCs is significantly affected, resulting in GSC spheres of uneven sizes and with their intercellular connections becoming looser and less close. In particular, the cells around GSC spheres stretched tentacles out, changing from round cells to long and narrow ones, similar to adherent cells. These results suggest that LIPUS inhibits the maintenance of GSC stem cell-related characteristics. The cell cycle and RNA sequence results showed that the key proteins at the $\mathrm{G} 2 / \mathrm{M}$ checkpoint were significantly upregulated and that the cell ratio in the G2/M phase was obviously increased in response to LIPUS treatment. This indicates that LIPUS promotes quiescent GSCs to transition from the $G 0$ phase to the $G 2 / M$ phase, to prepare proteins and other substances for mitosis.

Current chemoradiotherapy mainly targets tumor cells in the proliferative phase and plays a killing role during tumor cell division. However, chemoradiotherapy cannot kill CSCs because DNA replication stops in the G0 phase. Then, we speculated that LIPUS-treated GSCs would reduce chemoradiotherapy resistance and increase treatment efficacy. Our results showed that in LIPUS-treated GSCs, the stem cell characteristics became less obvious, while the sensitivity to the chemotherapy drug TMZ was increased and the drug resistance was reduced. These results suggested that compared with TMZ treatment, the volume of the tumors in response to LIPUS + TMZ treatment was diminished. Meanwhile, the results of TUNEL and Ki67 staining also suggested that LIPUS enhanced the cytotoxicity of TMZ, promoted the apoptosis of tumor cells, and inhibited the proliferation of tumor cells. It is clear that LIPUS promoted the inhibitory effect of TMZ on the biological activity of tumor cells and enhanced the sensitivity of subcutaneous U87 transplanted tumors to TMZ in vivo(30). LIPUS, as a physical means, can provide new ideas for clinical solutions to postoperative chemotherapy-drug resistance, improve the efficacy of chemotherapy drugs, and reduce tumor recurrence $(29,38,39)$. 
Cancer stem cells can have metabolic characteristics that set them apart from proliferating tumors. Although proliferative tumor cells rely on aerobic glycolysis, which is known as the Warburg effect, slowcycling cancer stem cells may prefer mitochondrial respiration as a primary source of energy $(17-19,40)$. The JC-1 results showed that LIPUS induced depolarization of the mitochondrial membrane potential and mitochondrial apoptosis and promoted oxidative stress. As the "energy factory" of cells, mitochondria are damaged and disrupted, producing a large amount of reactive oxygen species (ROS), which induces DNA and telomere damage $(41,42)$. Persistent DNA damage could aggravate mitochondrial disorders and release more ROS(43), thus inducing grave DNA damage. Moreover, it is difficult for telomeres to be repaired during regular DNA repair $(44,45)$. Telomeres are the "caps" that adorn the ends of human chromosomes, composed of TTAGGG repeated sequences. Telomeres are mainly involved in DNA repair, protect the ends of chromosomes, delay chromosome shortening, and maintain chromosome stability(22, $46,47)$. In addition, telomerase activity is upregulated to maintain the ability of proliferation and selfrenewal in germ cells and embryonic stem cells. Active telomerase is also one of the characteristics of cancer stem cells(48). The FISH and q-PCR results suggested that telomere length was significantly shortened and telomere deletion increased in response to LIPUS treatment. 53 bp 1 is a DNA repair protein for double-strand breaks, which is recruited to telomeres upon double-strand break formation. The IF-FISH results suggested that the number of 53BP1-positive telomere dysfunction-induced foci (TIFs) increased significantly after LIPUS, indicating a telomere crisis. Elise Fouquerel et al. reported that local singlet oxygen $\left({ }^{1} \mathrm{O}_{2}\right)$, a type of ROS, specifically induces guanine $(G)$ on telomeres to produce 8-oxoG, causing telomere damage(20,21). Moreover, 8-oxoG reduces DNA polymerase and telomerase activity, shortening telomeres. Our results indicated that with LIPUS treatment, ${ }^{1} \mathrm{O}_{2}$ production in GSCs was significantly increased and the telomeres were destroyed. Therefore, we found that LIPUS is related to telomere damage by ${ }^{1} \mathrm{O}_{2}$, the mechanism by which LIPUS inhibits the stemness expression of CSCs and promotes CSC expulsion from quiescence.

Sonosensitizers in tumor cells, such as hematoporphyrin, absorb energy and undergo electronic transitions after ultrasonic treatment, where they are excited from a low-energy state to a high-energy state, then transfer their energy to the ground state oxygen molecules to release ${ }^{1} \mathrm{O}_{2}$ and cause irreversible damage to cancer cells(49). Ma et al. successfully synthesized three metal 4methylphenylporphyrin complexes and encapsulated them with human serum albumin to form novel nanosonosensitizers(50). These nanosonosensitizers generated abundant ${ }^{1} \mathrm{O}_{2}$ in response to ultrasonic irradiation. The cytochromes (cyt) are proteins with characteristic strong absorption of visible light due to their iron-containing heme prosthetic groups. In human somatic cells, cytochromes widely exist in organelles and the cytoplasmic matrix. Among them, cyt $\mathrm{C} 1$ and cyt $\mathrm{C}$ both contain one heme, which is distributed in the inner mitochondrial membrane and is higher in metabolically active cells(51). Studies have shown that glioma cells have calcium overload and significantly increased ROS after ultrasound treatment. It is believed that the endoplasmic reticulum may be the main target of hemoporphyrin monomethyl ether-sonodynamic therapy(52). Cyt B5a and cytB5b both contain one heme and are distributed in the endoplasmic reticulum and mitochondria. Therefore, we suspect that LIPUS, as a form 
of physical energy, causes the heme prosthetic groups of the cyt C family and cyt B5 family in mitochondria to undergo an electronic transition to produce ${ }^{1} \mathrm{O}_{2}$. Notably, we found that LIPUS significantly elevated ${ }^{1} \mathrm{O}_{2}$ production in vitro in response to LIPUS stimulation of recombinant cyt $\mathrm{C}$, cyt $\mathrm{C} 1$, cyt $\mathrm{B} 5 \mathrm{a}$, and cyt $\mathrm{B} 5 \mathrm{~b}$ proteins, again confirming that the mitochondrial respiratory chain members cyt $\mathrm{C}$, cyt C1, cyt B5a and cyt B5b may be the targets for LIPUS energy conversion. We propose that cytochromes should be able to produce ${ }^{1} \mathrm{O}_{2}$ with different efficiencies under ultrasound irradiation. Next, we will compare the efficiency of ${ }^{1} \mathrm{O}_{2}$ generation by the cyt $\mathrm{C}$ family and cyt $\mathrm{B} 5$ family.

The previous literature generally believed that ultrasound induced sonosensitizers to produce ${ }^{1} \mathrm{O}_{2}$ due to acoustic cavitation and sonoluminescence(53-55), but this often requires a higher sound intensity beyond diagnostic dose ultrasound. Ultrasound, as a kind of material vibration wave, can form phonons at the level of protein macromolecules and cause phonon-electron coupling(56). Phonons change sonosensitizers from the ground state to the excited state, and then the excited sonosensitizers can convert triplet oxygen molecules (the main form of oxygen molecules in the air) into singlet states. Therefore, we believe that acoustic cavitation and sonoluminescence may not be its internal mechanism but instead that LIPUS causes phonon-electron coupling in protein macromolecules to induce electronic transitions and then improve ${ }^{1} \mathrm{O}_{2}$ generation. Currently, there are few results available to prove this viewpoint in this project. We will confirm it in future research. Currently, most researchers focus on the killing effect of ultrasound on tumors, while our experiment focused on the "waking up" effect of LIPUS on CSCs. In contrast to studies on sonodynamic therapy combined with synthetic sonosensitive agents,

we found that the cyt B5 and cyt $\mathrm{C}$ families with LIPUS and mitochondria increased intracellular ${ }^{1} \mathrm{O}_{2}$ production through energy transfer and electron transitions rather than through adding exogenous sonosensitive agents. ${ }^{1} \mathrm{O}_{2}$ induces telomere damage, shortens telomeres and promotes the differentiation of dormant CSCs into metabolically active tumor cells (Fig.6).

In summary, LIPUS awakens quiescent GSCs, enhances the cytotoxic effects of TMZ and increases TMZ sensitivity. It provides a new way to solve the problem of postoperative chemoradiotherapeutic drug tolerance in clinical glioma patients and avoids the damage to normal brain tissue caused by high field intensity ultrasound due to high energy and scattering. According to abundant experience in the application of low-field intensity ultrasound in clinical diagnosis(10), such as color Doppler ultrasonography for pregnant women, this proves that the biosafety of this energy range has been fully affirmed, which also provides reference experiences and application prospects for the treatment of "tumor stem cell wake-up" by repeated low-field intensity ultrasound.

\section{Conclusions}

In recent years, GSC has been a target for glioma therapy because of its association with tumorigenesis and drug resistance, and LIPUS promotes mesenchymal stem cells differentiation and is widely used in tissue engineering. We found that LIPUS "wakes quiescent GSCs up" and enhances the sensitivity of chemoradiotherapy to promote the killing role of chemoradiotherapeutic drugs on GSCs, providing new 
ideas for prolonging the survival of patients. In addition, as sonosensitizers, cytochrome B5 and C can absorb energy and undergo electronic transitions in response to LIPUS treatment, which are excited from a low-energy state to a high-energy state, and then transfer the energy to the ground state oxygen molecules to release singlet ${ }^{1} \mathrm{O}_{2}$. Then the released ${ }^{1} \mathrm{O}_{2}$ from the mitochondria leads to a telomere crisis and dysfunction.

\section{Abbreviations}

CSC: cancer stem cell; GCS: glioma stem cell; LIPUS: low-intensity pulsed ultrasound; CCK8: cell counting kit-8; FISH: fluorescence in situ hybridization; IF-FISH: Immunofluorescence and telomere fluorescence in situ hybridization; RT-PCR: Reverse transcription-polymerase chain reaction; qPCR: Quantitative polymerase chain reaction; ROS: reactive oxygen species; Cyt: cytochrome; FACS: Fluorescence activated Cell Sorting; ${ }^{1} \mathrm{O}_{2}$ : singlet oxygen; 7-AAD: 7-aminoactinomycin D; TIFs: telomere dysfunction-induced focus; TMZ: Temozolomide; TUNEL: TdT-mediated dUTP nick end labeling; BrdU: 5-bromodeoxyuridine; $\mathrm{ddH}_{2} \mathrm{O}$ : deionized distilled water; $\mathrm{RQ}$ : fold-change

\section{Declarations}

Ethics approval and consent to participate『Not applicable.

Consent for publication[Not applicable.

Availability of data and materials₫Not applicable.

Competing interests: The authors declare that they have no competing interests.

Funding: This research was supported by the grants awarded by Natural Science Foundation of Tianjin Municipal Science and Technology Commission (20JCQNJC00410) and National Natural Science Foundation of China (81871029).

Author contributions: Hua Yan and Xiaoguang Tong designed the experiments. Glioma stem cells were cultured by Sirong Song and Qiong Wang. FASC, RT-PCR, qPCR and western blotting experiments and data analysis were performed by Lixia $\mathrm{Xu}$ and Sirong Song. Mice were generated and experiments on LIPUS promoting TMZ sensitivity were carried out by Dongbin Ma and Lanxiang Liu. Sirong Song performed and analyzed the rest experiments. Sirong Song and Lixia Xu wrote and edited the manuscript.

Acknowledgements: We would like to thank the Department of Ultrasound at Tianjin Huanhu Hospital for providing the ultrasound machine.

\section{References}


1. Nusblat LM, Carroll MJ, Roth CM. Crosstalk between M2 macrophages and glioma stem cells. Cell Oncol (Dordr). 2017;40(5):471-82.

2. Lathia JD, Mack SC, Mulkearns-Hubert EE, Valentim CL, Rich JN. Cancer stem cells in glioblastoma. Genes Dev. 2015;29(12):1203-17.

3. Clevers H. The cancer stem cell: premises, promises and challenges. Nat Med. 2011;17(3):313-9.

4. Matteucci C, Balestrieri E, Argaw-Denboba A, Sinibaldi-Vallebona P. Human endogenous retroviruses role in cancer cell stemness. Semin Cancer Biol. 2018;53:17-30.

5. Reya T, Morrison SJ, Clarke MF, Weissman IL. Stem cells, cancer, and cancer stem cells. Nature. 2001;414(6859):105-11.

6. Bao S, Wu Q, McLendon RE, Hao Y, Shi Q, Hjelmeland AB, et al. Glioma stem cells promote radioresistance by preferential activation of the DNA damage response. Nature. 2006;444(7120):75660.

7. Najafi M, Mortezaee K, Majidpoor J. Cancer stem cell (CSC) resistance drivers. Life Sci. 2019;234:116781.

8. Zhou BB, Zhang H, Damelin M, Geles KG, Grindley JC, Dirks PB. Tumour-initiating cells: challenges and opportunities for anticancer drug discovery. Nat Rev Drug Discov. 2009;8(10):806-23.

9. Clarke MF, Dick JE, Dirks PB, Eaves CJ, Jamieson CH, Jones DL, et al. Cancer stem cells-perspectives on current status and future directions: AACR Workshop on cancer stem cells. Cancer Res. 2006;66(19):9339-44.

10. Mayo PH, Copetti R, Feller-Kopman D, Mathis G, Maury E, Mongodi S, et al. Thoracic ultrasonography: a narrative review. Intensive Care Med. 2019;45(9):1200-11.

11. Wang X, Jia Y, Wang P, Liu Q, Zheng H. Current status and future perspectives of sonodynamic therapy in glioma treatment. Ultrason Sonochem. 2017;37:592-9.

12. Harrison A, Lin S, Pounder N, Mikuni-Takagaki Y. Mode \& mechanism of low intensity pulsed ultrasound (LIPUS) in fracture repair. Ultrasonics. 2016;70:45-52.

13. Armstrong JPK, Puetzer JL, Serio A, Guex AG, Kapnisi M, Breant A, et al. Engineering Anisotropic Muscle Tissue using Acoustic Cell Patterning. Adv Mater. 2018;30(43):e1802649.

14. Alexandrov AV. Ultrasound enhanced thrombolysis for stroke. Int J Stroke. 2006;1(1):26-9.

15. Ni XJ, Wang XD, Zhao YH, Sun HL, Hu YM, Yao J, et al. The Effect of Low-Intensity Ultrasound on Brain-Derived Neurotropic Factor Expression in a Rat Sciatic Nerve Crushed Injury Model. Ultrasound Med Biol. 2017;43(2):461-8.

16. Costa V, Carina V, Fontana S, De Luca A, Monteleone F, Pagani S, et al. Osteogenic commitment and differentiation of human mesenchymal stem cells by low-intensity pulsed ultrasound stimulation. $J$ Cell Physiol. 2018;233(2):1558-73.

17. Viale A, Draetta GF. Metabolic Features of Cancer Treatment Resistance. Recent Results Cancer Res. 2016;207:135-56. 
18. Caro P, Kishan AU, Norberg E, Stanley IA, Chapuy B, Ficarro SB, et al. Metabolic signatures uncover distinct targets in molecular subsets of diffuse large B cell lymphoma. Cancer Cell. 2012;22(4):54760.

19. Cole A, Wang Z, Coyaud E, Voisin V, Gronda M, Jitkova Y, et al. Inhibition of the Mitochondrial Protease ClpP as a Therapeutic Strategy for Human Acute Myeloid Leukemia. Cancer Cell. 2015;27(6):864-76.

20. Qian W, Kumar N, Roginskaya V, Fouquerel E, Opresko PL, Shiva S, et al. Chemoptogenetic damage to mitochondria causes rapid telomere dysfunction. Proc Natl Acad Sci U S A. 2019;116(37):18435-44.

21. Fouquerel E, Barnes RP, Uttam S, Watkins SC, Bruchez MP, Opresko PL. Targeted and Persistent 8Oxoguanine Base Damage at Telomeres Promotes Telomere Loss and Crisis. Mol Cell. 2019;doi: 10.1016/j.molcel.2019.04.024

22. Laprade H, Querido E, Smith MJ, Guerit D, Crimmins H, Conomos D, et al. Single-Molecule Imaging of Telomerase RNA Reveals a Recruitment-Retention Model for Telomere Elongation. Mol Cell. 2020;doi: 10.1016/j.molcel.2020.05.005.

23. Rudolph KL, Chang S, Lee HW, Blasco M, Gottlieb GJ, Greider C, et al. Longevity, stress response, and cancer in aging telomerase-deficient mice. Cell. 1999;96(5):701-12.

24. Valent P, Bonnet D, De Maria R, Lapidot T, Copland M, Melo JV, et al. Cancer stem cell definitions and terminology: the devil is in the details. Nat Rev Cancer. 2012;12(11):767-75.

25. Saygin C, Matei D, Majeti R, Reizes O, Lathia JD. Targeting Cancer Stemness in the Clinic: From Hype to Hope. Cell Stem Cell. 2019;24(1):25-40.

26. Auffinger B, Tobias AL, Han Y, Lee G, Guo D, Dey M, et al. Conversion of differentiated cancer cells into cancer stem-like cells in a glioblastoma model after primary chemotherapy. Cell Death Differ. 2014;21(7):1119-31.

27. Ting CY, Fan CH, Liu HL, Huang CY, Hsieh HY, Yen TC, et al. Concurrent blood-brain barrier opening and local drug delivery using drug-carrying microbubbles and focused ultrasound for brain glioma treatment. Biomaterials. 2012;33(2):704-12.

28. Guzman ML, Rossi RM, Karnischky L, Li X, Peterson DR, Howard DS, et al. The sesquiterpene lactone parthenolide induces apoptosis of human acute myelogenous leukemia stem and progenitor cells. Blood. 2005;105(11):4163-9.

29. Qu F, Wang P, Zhang K, Shi Y, Li Y, Li C, et al. Manipulation of Mitophagy by "All-in-One" nanosensitizer augments sonodynamic glioma therapy. Autophagy. 2020;16(8):1413-35.

30. Wu P, Dong W, Guo X, Qiao X, Guo S, Zhang L, et al. ROS-Responsive Blended Nanoparticles: Cascade-Amplifying Synergistic Effects of Sonochemotherapy with On-demand Boosted Drug Release During SDT Process. Adv Healthc Mater. 2019;8(18):e1900720.

31. Li YJ, Huang P, Jiang CL, Jia de X, Du XX, Zhou JH, et al. Sonodynamically induced anti-tumor effect of 5-aminolevulinic acid on pancreatic cancer cells. Ultrasound Med Biol. 2014;40(11):2671-9.

32. Harder BG, Blomquist MR, Wang J, Kim AJ, Woodworth GF, Winkles JA, et al. Developments in BloodBrain Barrier Penetrance and Drug Repurposing for Improved Treatment of Glioblastoma. Front 
Oncol. 2018;8:462.

33. Ning GZ, Song WY, Xu H, Zhu RS, Wu QL, Wu Y, et al. Bone marrow mesenchymal stem cells stimulated with low-intensity pulsed ultrasound: Better choice of transplantation treatment for spinal cord injury: Treatment for SCI by LIPUS-BMSCs transplantation. CNS Neurosci Ther. 2019;25(4):496508.

34. Xu ZY, Li XQ, Chen S, Cheng Y, Deng JM, Wang ZG. Glioma stem-like cells are less susceptible than glioma cells to sonodynamic therapy with photofrin. Technol Cancer Res Treat. 2012;11(6):615-23.

35. Wang X, Prager BC, Wu Q, Kim LJY, Gimple RC, Shi Y, et al. Reciprocal Signaling between Glioblastoma Stem Cells and Differentiated Tumor Cells Promotes Malignant Progression. Cell Stem Cell. 2018;doi: doi: 10.1016/j.stem.2018.03.011.

36. Mizuno H, Spike BT, Wahl GM, Levine AJ. Inactivation of p53 in breast cancers correlates with stem cell transcriptional signatures. Proc Natl Acad Sci U S A. 2010;107(52):22745-50.

37. Ishiguro T, Ohata H, Sato A, Yamawaki K, Enomoto T, Okamoto K. Tumor-derived spheroids: Relevance to cancer stem cells and clinical applications. Cancer Sci. 2017;108(3):283-9.

38. Wan Q, Zou C, Hu D, Zhou J, Chen M, Tie C, et al. Imaging-guided focused ultrasound-induced thermal and sonodynamic effects of nanosonosensitizers for synergistic enhancement of glioblastoma therapy. Biomater Sci. 2019;7(7):3007-15.

39. Trendowski M, Wong V, Zoino JN, Christen TD, Gadeberg L, Sansky M, et al. Preferential enlargement of leukemia cells using cytoskeletal-directed agents and cell cycle growth control parameters to induce sensitivity to low frequency ultrasound. Cancer Lett. 2015;360(2):160-70.

40. Viale A, Pettazzoni P, Lyssiotis CA, Ying H, Sanchez N, Marchesini M, et al. Oncogene ablationresistant pancreatic cancer cells depend on mitochondrial function. Nature. 2014;514(7524):628-32.

41. Liu J, Cao L, Chen J, Song S, Lee IH, Quijano C, et al. Bmi1 regulates mitochondrial function and the DNA damage response pathway. Nature. 2009;459(7245):387-92.

42. Passos JF, Saretzki G, von Zglinicki T. DNA damage in telomeres and mitochondria during cellular senescence: is there a connection? Nucleic Acids Res. 2007;35(22):7505-13.

43. Vousden KH, Lane DP. p53 in health and disease. Nat Rev Mol Cell Biol. 2007;8(4):275-83.

44. Ozer O, Bhowmick R, Liu Y, Hickson ID. Human cancer cells utilize mitotic DNA synthesis to resist replication stress at telomeres regardless of their telomere maintenance mechanism. Oncotarget. 2018;9(22):15836-46.

45. Ozer O, Hickson ID. Pathways for maintenance of telomeres and common fragile sites during DNA replication stress. Open Biol. 2018;8(4).

46. Tarry-Adkins JL, Martin-Gronert MS, Chen JH, Cripps RL, Ozanne SE. Maternal diet influences DNA damage, aortic telomere length, oxidative stress, and antioxidant defense capacity in rats. FASEB J. 2008;22(6):2037-44.

47. Mendez-Bermudez A, Giraud-Panis MJ, Ye J, Gilson E. Heterochromatin replication goes hand in hand with telomere protection. Nat Struct Mol Biol. 2020;27(4):313-8. 
48. Varela E, Munoz-Lorente MA, Tejera AM, Ortega S, Blasco MA. Generation of mice with longer and better preserved telomeres in the absence of genetic manipulations. Nat Commun. 2016;7:11739.

49. Zhang H, Chen J, Zhu X, Ren Y, Cao F, Zhu L, et al. Ultrasound induced phase-transition and invisible nanobomb for imaging-guided tumor sonodynamic therapy. J Mater Chem B. 2018;6(38):6108-21.

50. Ma A, Chen H, Cui Y, Luo Z, Liang R, Wu Z, et al. Metalloporphyrin Complex-Based Nanosonosensitizers for Deep-Tissue Tumor Theranostics by Noninvasive Sonodynamic Therapy. Small. 2019;15(5):e1804028.

51. Daijima Y, Komatsu T. Haemoglobin wrapped covalently by human serum albumin mutants containing Mn(III) protoporphyrin IX: an $\mathrm{O} 2$ complex stable in H2O2 solution. Chem Commun (Camb). 2014;50(94):14716-9.

52. Li JH, Yue W, Huang Z, Chen ZQ, Zhan Q, Ren FB, et al. Calcium overload induces C6 rat glioma cell apoptosis in sonodynamic therapy. Int J Radiat Biol. 2011;87(10):1061-6.

53. Dezhkunov NV, Francescutto A, Ciuti P, Mason TJ, lernetti G, Kulak Al. Enhancement of sonoluminescence emission from a multibubble cavitation zone. Ultrason Sonochem. 2000;7(1):1924.

54. Sazgarnia A, Shanei A, Eshghi H, Hassanzadeh-Khayyat M, Esmaily H, Shanei MM. Detection of sonoluminescence signals in a gel phantom in the presence of Protoporphyrin IX conjugated to gold nanoparticles. Ultrasonics. 2013;53(1):29-35.

55. Dezhkunov NV, Francescutto A, Serpe L, Canaparo R, Cravotto G. Sonoluminescence and acoustic emission spectra at different stages of cavitation zone development. Ultrason Sonochem. 2018;40:104-9.

56. Viani L, Corbella M, Curutchet C, O'Reilly EJ, Olaya-Castro A, Mennucci B. Molecular basis of the exciton-phonon interactions in the PE545 light-harvesting complex. Phys Chem Chem Phys. 2014;16(30):16302-11.

\section{Table}

Table 1: qPCR and RT-PCR primer sequence 


\begin{tabular}{|ll|}
\hline Gene & Primer sequence \\
\hline Tel-F & GGTTTTTGAGGGTGAGGGTGAGGGTGAGGGTGAGGGT \\
\hline Tel-R & TCCCGACTATCCCTATCCCTATCCCTATCCCTATCCCT \\
\hline 36B4-R & CAGCAAGTGGGAAGGTGTAATCC \\
\hline human Nanog-F & AGTCCCATTCTATCATCAACGGGTACAA \\
\hline human Nanog-R & TGCTGGAGGCTGAGGTATTTCTGTCTC \\
\hline human OCT4-F & GACAGGGGGAGGGGAGGAGCTAGG \\
\hline human OCT4-R & CTTCCCTCCAACCAGTTGCCCCAAAC \\
\hline human sox2-F & GGGAAATGGGAGGGGTGCAAAAGAGG \\
\hline human sox2-R & TTGCGTGAGTGTGGATGGGATTGGTG \\
\hline human CD133 F & CAGAGTACAACGCCAAACCA \\
\hline human CD133 R & AAATCACGATGAGGGTCAGC \\
\hline human nestin F & GAAACAGCCATAGAGGGCAAA \\
\hline human nestin R & TGGTTTTCCAGAGTCTTCAGTGA \\
\hline human $\beta$-catin F & CTCCATCCTGGCCTCGCTGT \\
\hline human $\beta$-catin R & GCTCGTACCTTCACCGTTCC \\
\hline
\end{tabular}

\section{Figures}




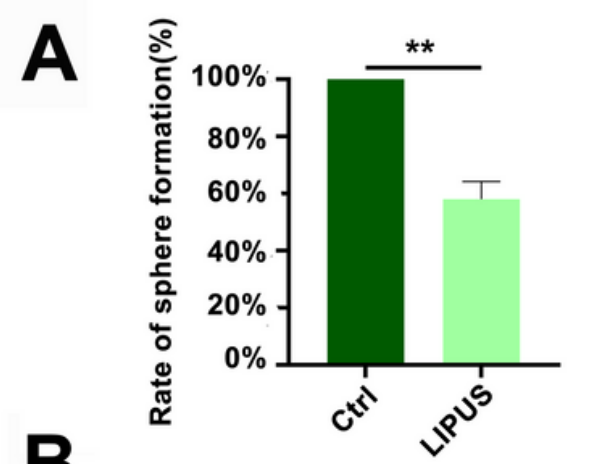

\section{Ctrl}
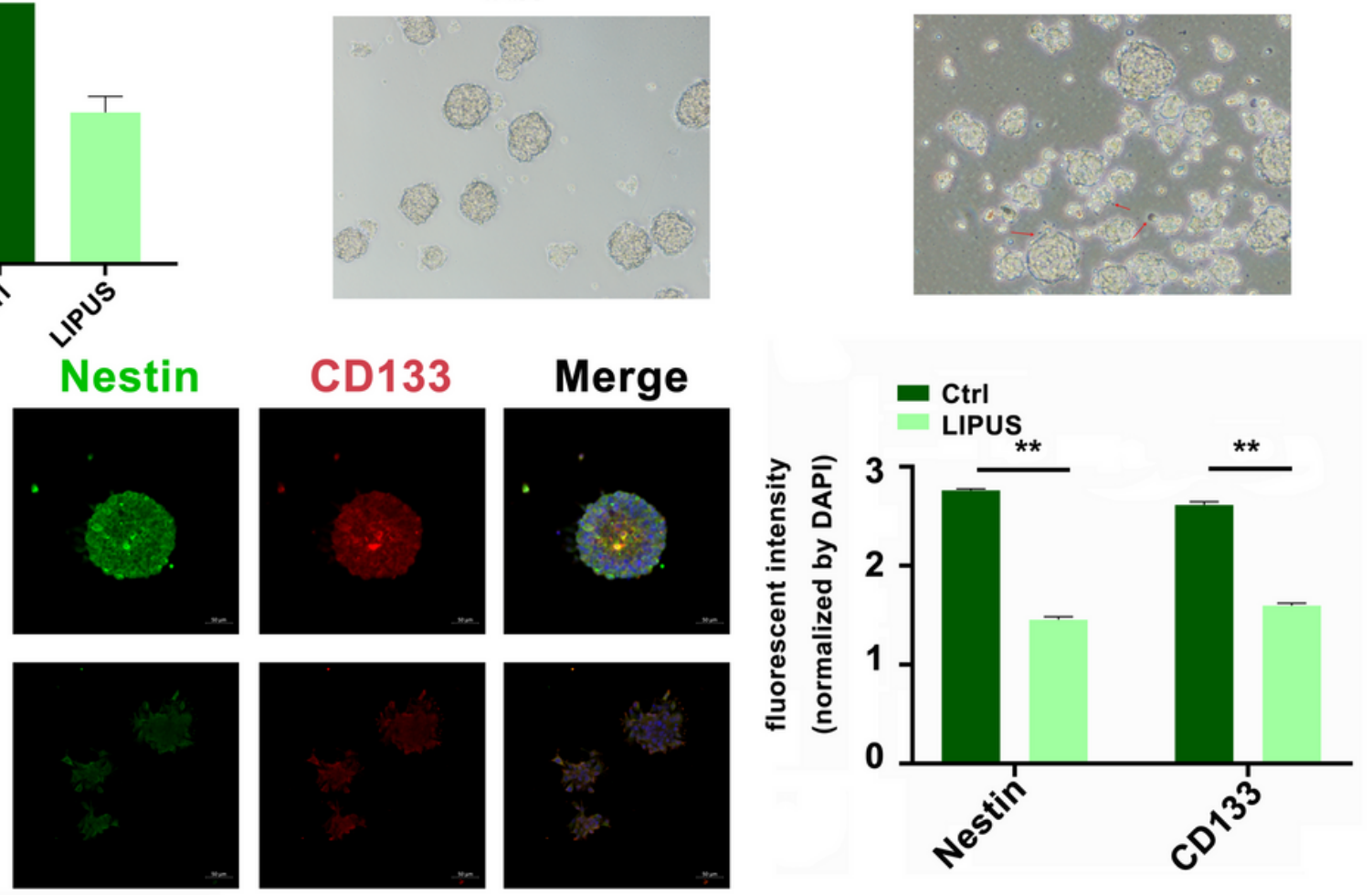

C

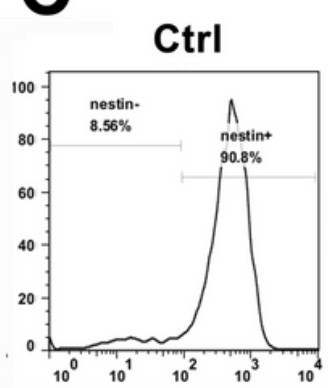

D
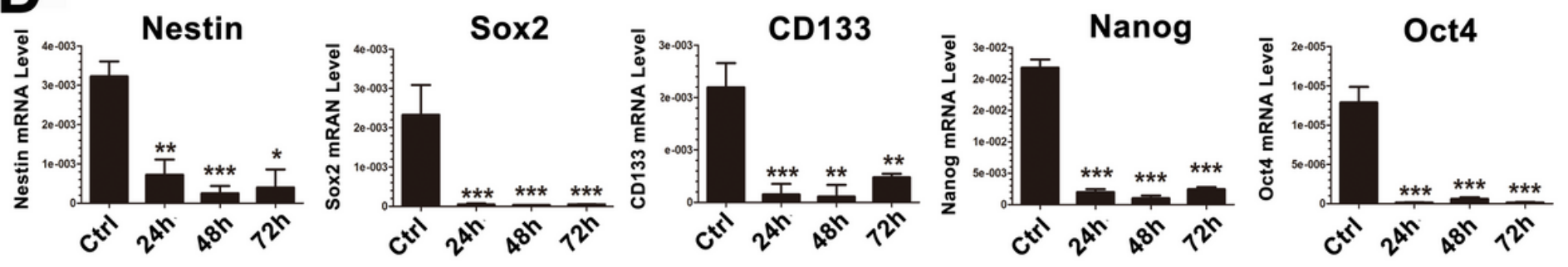

\section{Figure 1}

LIPUS promotes GSCs to escape from the stem cell state. (A)The GSC sphere formation ratio, showing the ability of sphere formation of GSCs. Representative images of glioma stem cell (GSC) spheres from U87 and GSC spheres with LIPUS treatment. Red arrowhead for GSC spheres with extended antennae. The data are expressed as the means \pm SD. ${ }^{* \star} p<0.01$, one-way ANOVA. (B) Representative images of GSC spheres expressed stem cell markers Nestin (green) and CD133 (red) by immunofluorescence. DAPI (blue) for nuclei. Quantification of stem cell markers signal intensity were normalized by DAPI. The data are 
expressed as the means $\pm S D$. ${ }^{*}$ p $<0.01$, one-way ANOVA. (C) Nestin expression with LIPUS treatment in $24 \mathrm{~h}, 48 \mathrm{~h}$ and $72 \mathrm{~h}$ and Nestin-negative cell ratio by flow cytometry. The data are expressed as the means \pm SD. ${ }^{*} p<0.01$, one-way ANOVA. (D) RT-PCR analysis of Nestin, Sox2, CD133, Nanog and Oct4 mRNA expression in GSCs with LIPUS treatment in $24 \mathrm{~h}, 48 \mathrm{~h}$ and $72 \mathrm{~h}$ respectively by RT-PCR. The data are expressed as the means $\pm S D$. ${ }^{*} p<0.05$, $* * p<0.01$, ${ }^{* * *} p<0.001$, one-way ANOVA.

A
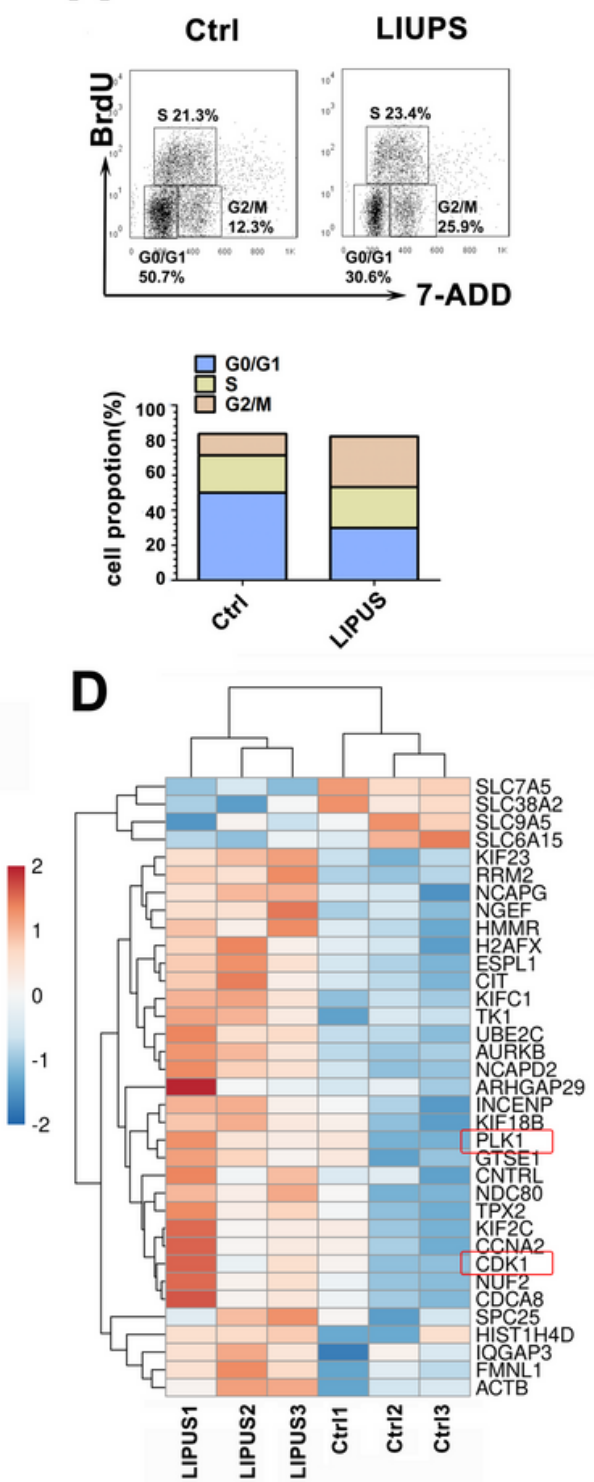

B

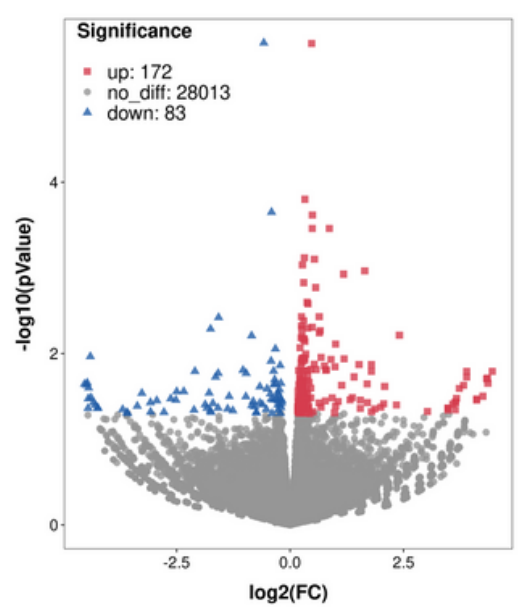

E

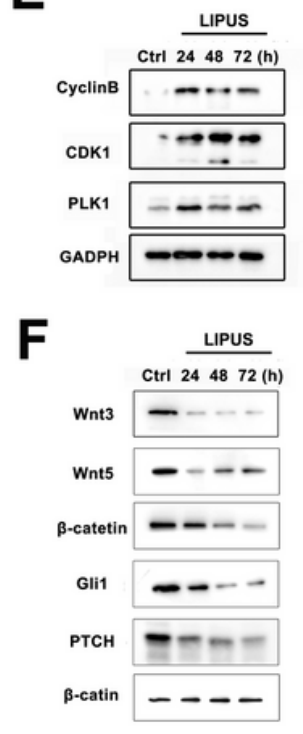

C

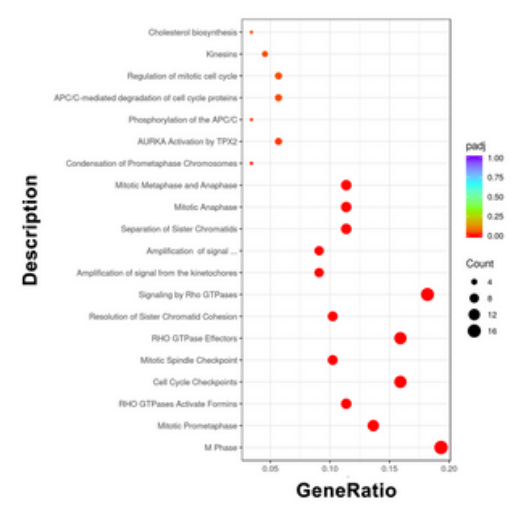

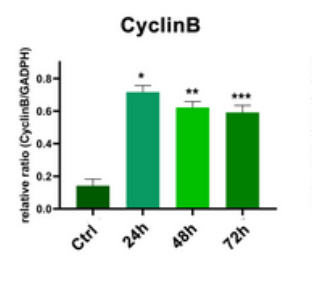
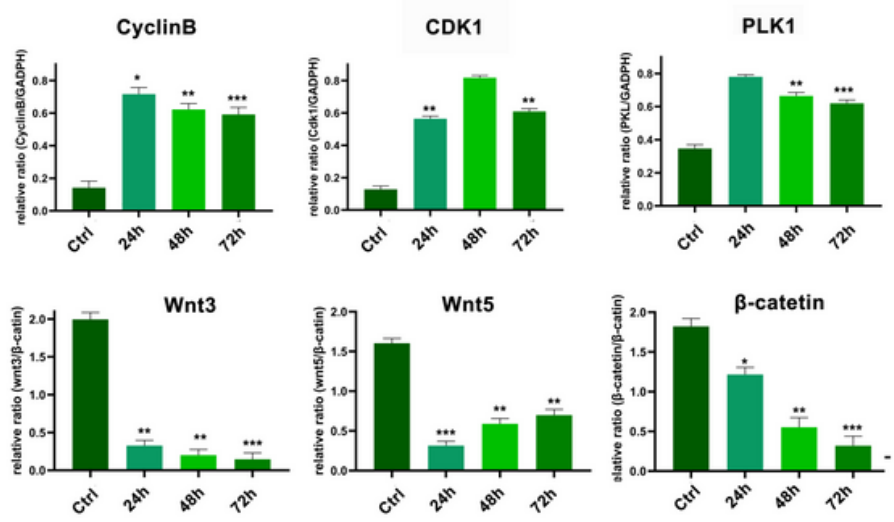

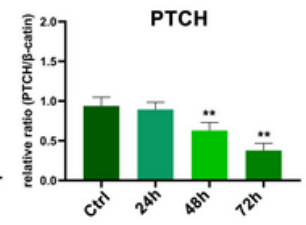

\section{Figure 2}

LIPUS regulates the G2/M checkpoint and reduces stem cell characteristics of GSC. (A) The BrdU and 7AAD analysis of GSCs with LIPUS treatment by flow cytometry. (B) The volcano plot of different gene between Ctrl and LIPUS by RNA-Seq. (C) The top 20 significantly enriched Reactome pathways of GSC with LIPUS treatment. (D) The cluster heat map of expression patterns of upregulated (red) and downregulated (blue) genes of GSC with LIPUS treatment. (E) Western blotting analysis expression of the key proteins of the G2/M checkpoint, including CyclinB, CDK1 and PLK1. The results (means \pm SD. ) 
represent ratios of each protein and GAPDH levels, and are normalized to Ctrl. (F) Western blotting analysis expression of the key nodes of the Hedgehog $(\mathrm{Hh})$ and Wnt/ $\beta$-catenin pathways, including Wnt3, Wnt5, $\beta$-catenin, Gli1 and PTCH. The results (means \pm SD. ) represent ratios of each protein and $\beta$-catin levels, and are normalized to Ctrl. The data are expressed as the means $\pm S D$. ${ }^{*} p<0.05,{ }^{*} p<0.01$, $\star * * p<0.001$, one-way ANOVA.

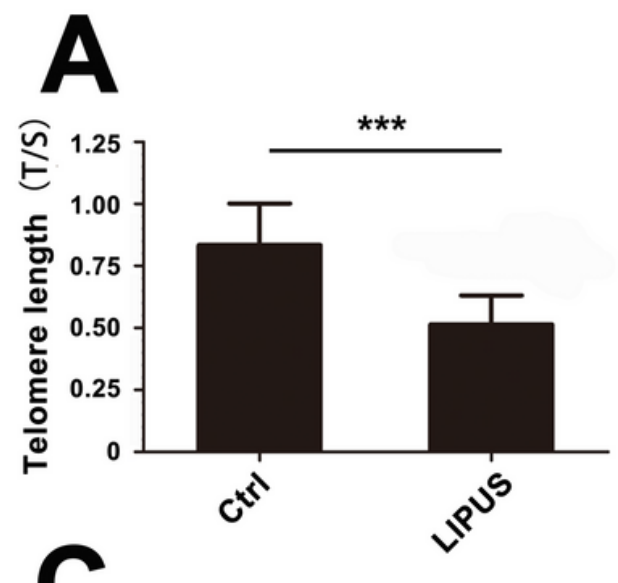

DAPI
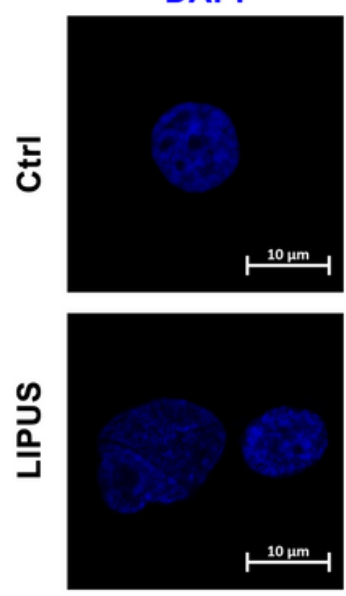

Telomere
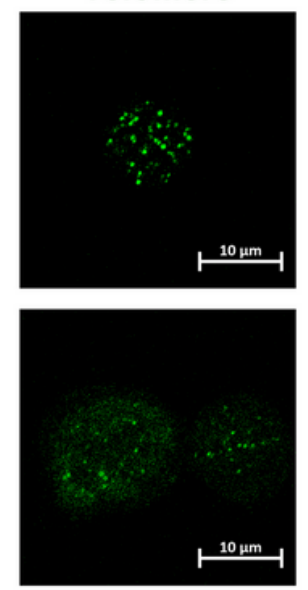
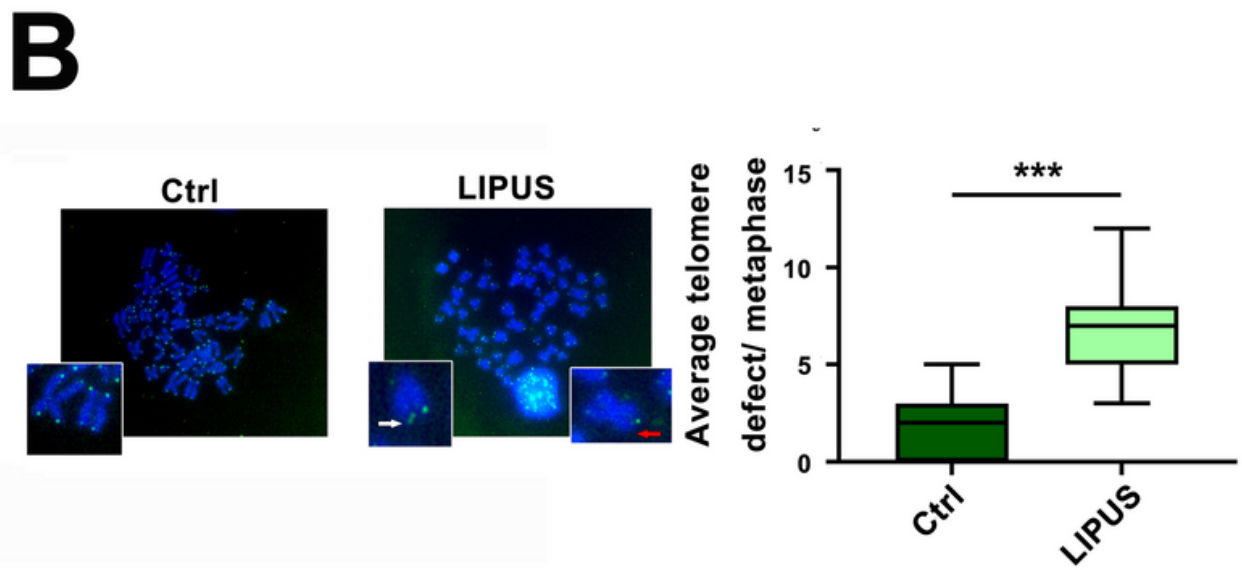

53bp1
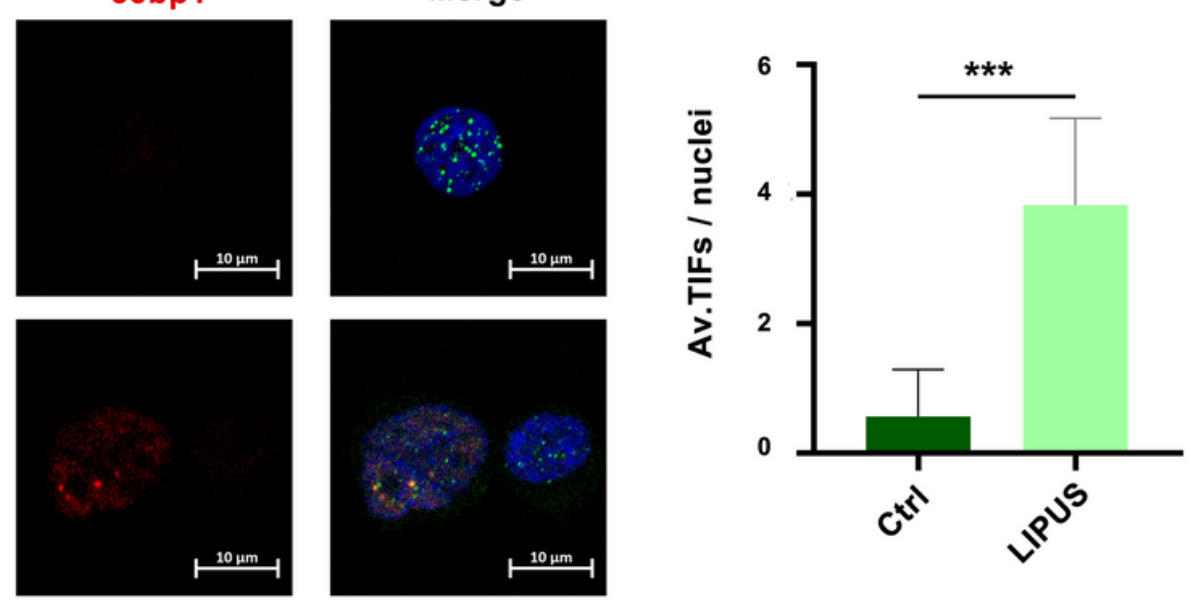

Figure 3

LIPUS shortens the telomere length and leads to telomere damage. (A) qPCR analysis of the telomere length (T/S) in GSCs with LIPUS treatment. The data are expressed as the means \pm SD. ${ }^{* *} p<0.001$, oneway ANOVA. (B) Representative images of Telomere metaphase spread and telomere fluorescence in situ hybridization analysis in GSCs with LIPUS treatment (red arrowheads telomeric loss and white arrowheads for fragile telomeres). Quantification of incomplete telomere. Each dot for a metaphase. Medians (bar) from 3 independent experiments (30 metaphases per condition for each experiment). The data are expressed as the means \pm SD. ${ }^{* *} p<0.001$, one-way ANOVA. (C) Representative images of immunofluorescence and telomere fluorescence in situ hybridization of TIFs in GSCs with LIPUS treatment (red for 53bp1 and green for telomeres). Quantification of the average (av.) number of TIFs per nuclei. Means \pm SD. of 3 independent experiments, at least 100 cells per experiment. The data are expressed as the means $\pm S D$. ${ }^{* *} p<0.001$, one-way ANOVA. 

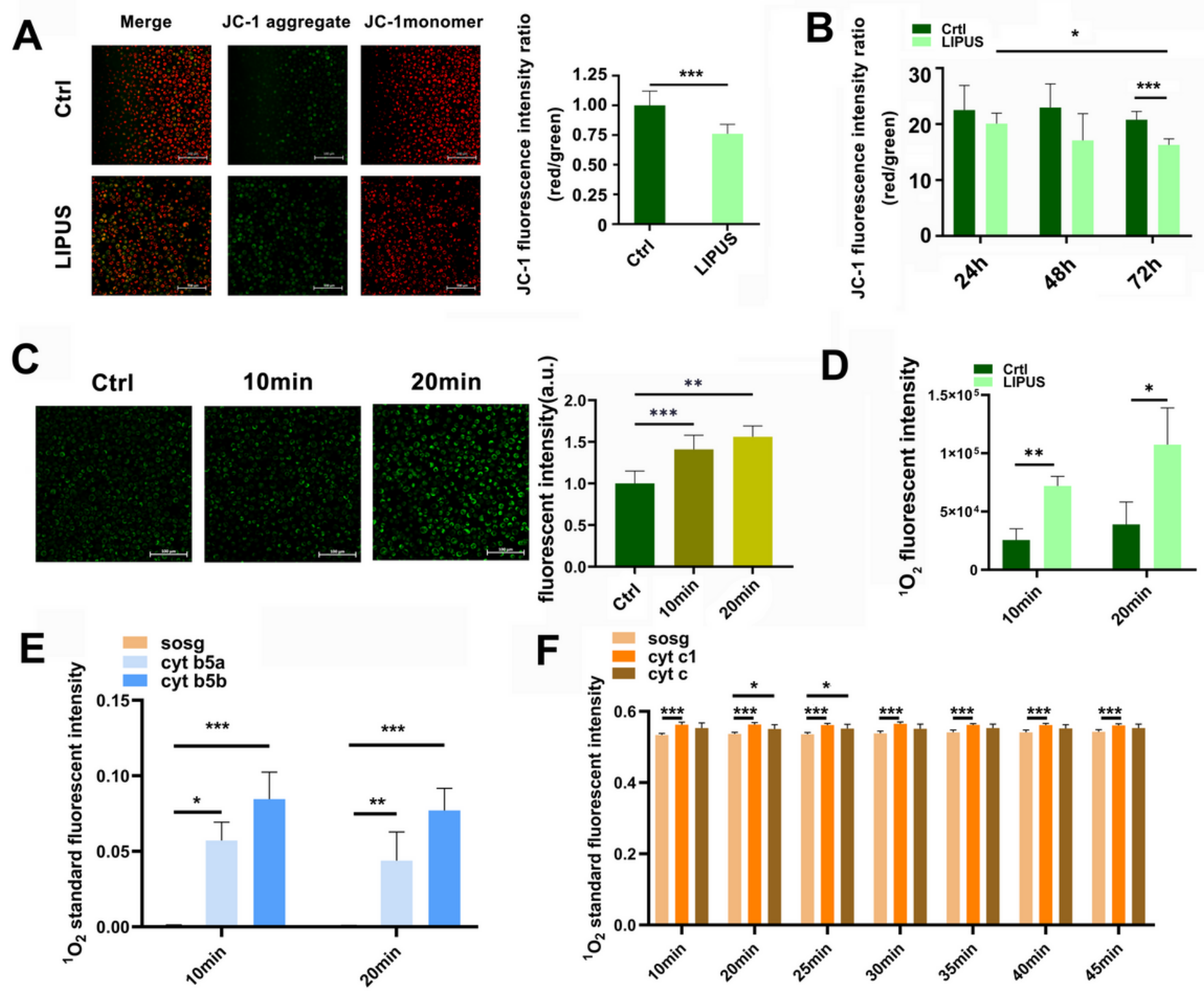

\section{Figure 4}

LIPUS on mitochondrial cytochrome (cyt) improves singlet oxygen (102) generation. (A) Representative images of Mitochondrial membrane potential analysis of GSCs with LIPUS treatment. The results of quantified fluorescence images are normalized to Ctrl. The data are expressed as the means \pm SD. $\star \star \star p<0.001$, one-way ANOVA. (B) JC-1 fluorescence intensity of GSCs with LIPUS treatment by a enzyme labeler. The data are expressed as the means $\pm S D$. ${ }^{*} p<0.05,{ }^{*} *{ }^{*}<0.001$, one-way ANOVA. (C) Representative images of 102 generation in GSCs with LIPUS treatment for 10 min and 20 min. The results of quantified fluorescence images are normalized to Ctrl. The data are expressed as the means \pm SD. ${ }^{*} p<0.01,{ }^{*} * \mathrm{p}<0.001$, one-way ANOVA. (D) 102 generation analysis in GSCs by an enzyme labeler. The data are expressed as the means \pm SD. ${ }^{*} p<0.05$, ${ }^{*} p<0.01$, one-way ANOVA. (E and F) 102 generation analysis in recombinant cyt B5a and cyt B5b with LIPUS treatment for 10 min and 20 min and recombinant cyt $\mathrm{C}$ and cyt $\mathrm{C} 1$ with LIPUS treatment for $10 \mathrm{~min}, 20 \mathrm{~min}, 25 \mathrm{~min}, 30 \mathrm{~min}, 35 \mathrm{~min}, 40 \mathrm{~min}$ 
and 45 min respectively by a enzyme labeler. The data are expressed as the means $\pm S D$. ${ }^{*} p<0.05$, ${ }^{* *} p<0.01, * \star * p<0.001$, one-way ANOVA.
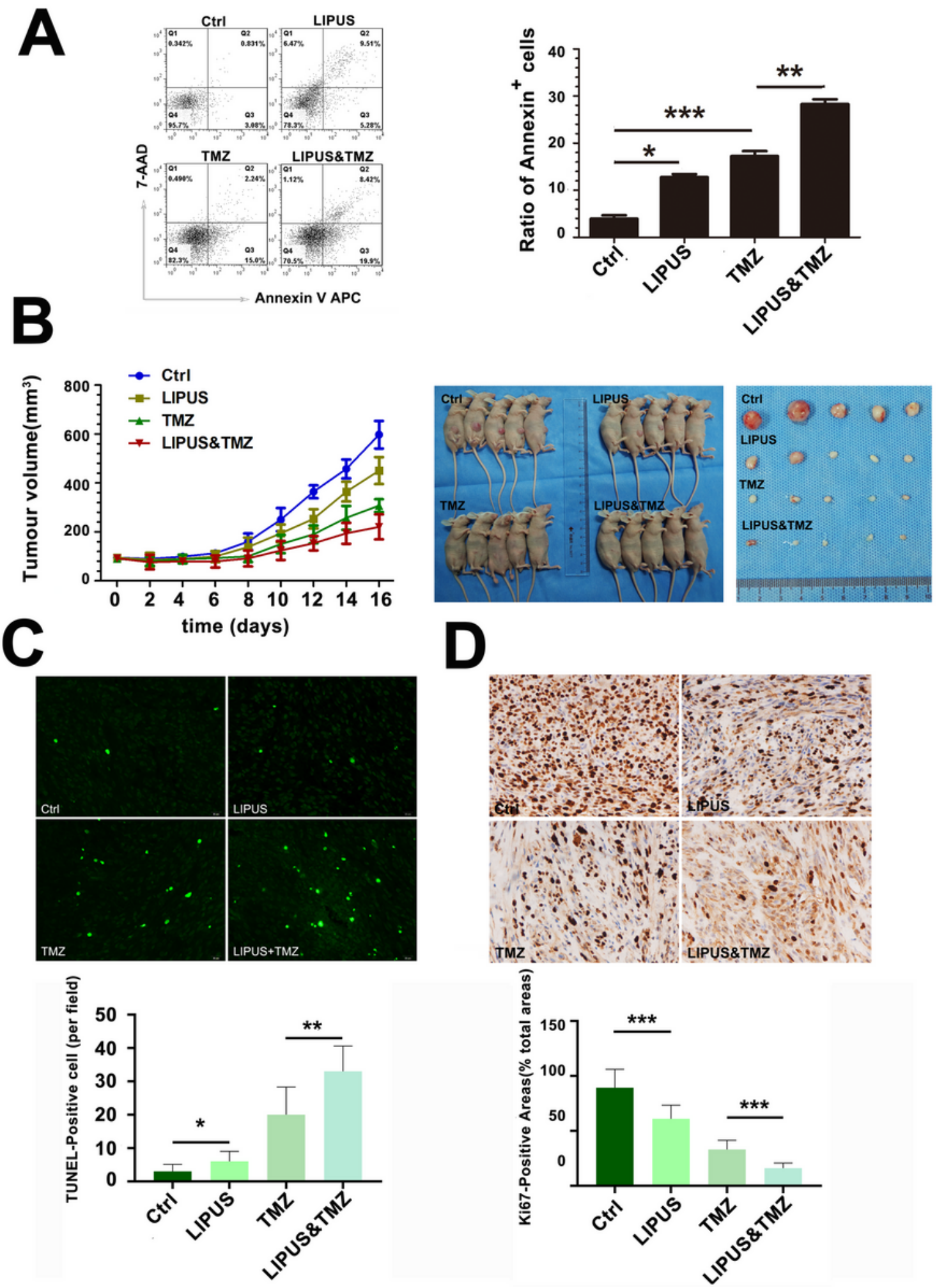

Figure 5

LIPUS enhances the chemotherapeutic sensitivity of TMZ and increases the cytotoxic effect on GSCs. (A) Annexin V-APC/7-AAD assay of GSCs with LIPUS treatment by flow cytometry. The data are expressed as the means \pm SD. ${ }^{*} p<0.05,{ }^{* *} p<0.01,{ }^{* * *} p<0.001$, one-way ANOVA. (B) The longest diameter (a) and the 
shortest diameter (b) of the tumor nodules measured with Vernier calipers daily. The tumor volume was calculated according to the formula $V=1 / 6 \pi(a b 2)$. (C) Representative images of TUNEL assay for the apoptosis level of tumor tissues. The data are expressed as the means $\pm S D$. ${ }^{*} p<0.05,{ }^{*} p<<0.01$, one-way ANOVA. D. Representative images of Ki67 assay for the proliferation level of tumor tissues. The data are expressed as the means $\pm S D$. ${ }^{* *} p<0.001$, one-way ANOVA.

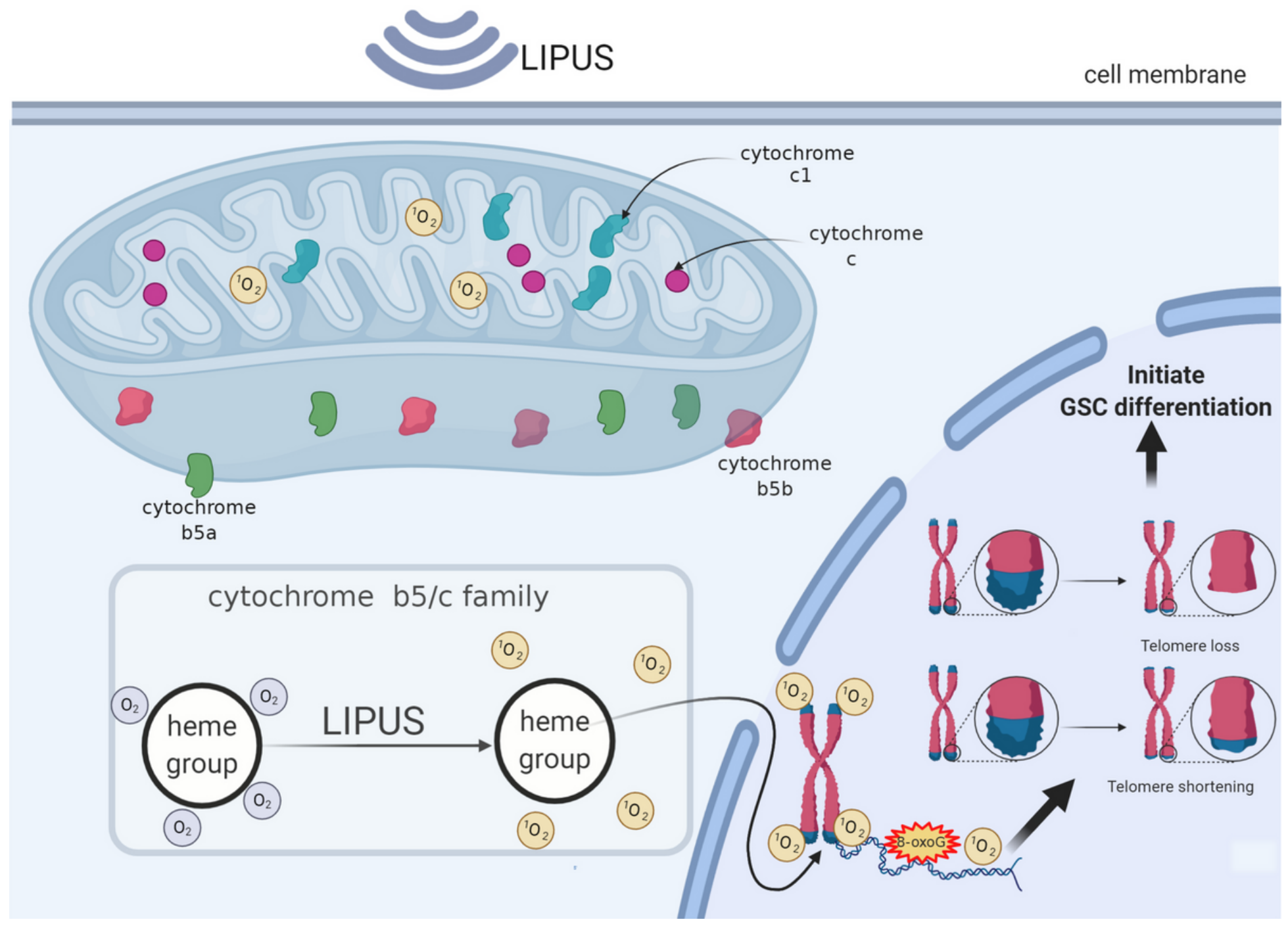

Figure 6

Schematic representation of the processes in which LIPUS-produced-102 leads to GSC differentiation. After LIPUS treatment, the heme groups in the mitochondrial cytochrome b5/c family absorb energy and are excited from a low-energy state to a high-energy state, then transfer their energy to the ground state oxygen molecules to release 102. The released 102 targets the 8-oxoguanine of telomeres, resulting in telomere shortening and loss, which is necessary to initiate GSC differentiation. 